\title{
Structural Topology Optimization Research for A Six-DOF Space Robotic Manipulator
}

\author{
Songbo Deng, Yan Wang, He Cai, Ke Li, Fan Yang, Yanbo Wang \\ Beijing Research Institute of Precise Mechatronics and Controls, No.1 South Da Hongmen Road, \\ Dong Gaodi, Feng Tai District, Beijing 100076, China
}

Keywords: space robotic manipulator, arm rod, light weight, multi constrained topology, finite element

\begin{abstract}
This paper focuses on the lightweight design of the 6-DOF space manipulator designed and assembled independently. The mass ratio of the arm rod is large which matters a lot on the positioning accuracy of the manipulator end. The light weight analysis is carried on to the arm rod of the space robotic manipulator. The stiffness and the natural frequency of each orders are taken as the constrain conditions so called the multi-constrained topology optimization. The optimized iterative design is made with BESO, and the optimized iterative speed is improved by the GBESO way. By comparison of the optimization data, the result shows that the stiffness and natural frequency of the manipulator will not be reduced while the mass of the arm rod is reduced. It proves the effectiveness of the optimization. This method could be applied to the design and optimization of other space manipulator.
\end{abstract}

\section{Introduction}

Space robotic manipulator is an indispensable tool for manned spaceflight, deep space exploration, space attack and defense, etc. It undertakes tasks such as grabbing, transferring, collecting and controlling spacecraft. In order to improve the flexibility, maneuverability and grasping performance of the space manipulator, the key of technology is to make the space manipulator more compact and lightweight. Some international research institute has conducted an in-depth study of the space robotic manipulator, including the MSS(Mobile Servicing System) of The Canadian international space station which is made up of MBS (Mobile Remote Servicer Base System), SSRMS (Space Station Remote Manipulator System) and SPDM (Special Purpose Dexterous Manipulator) [1]; FTS(Flight Telerobotic Servicer) [2]; Skyworker [3]; Robonaut [4]; ORBITAL EXPRESS of America [5]; JEMRMS (Remote Manipulator System) of Japan; ETS-VII as well as European ERA(The European Robotic Arm) of European. The arm of the manipulator is used to support and drag the rotating parts of the robot arm, which has a great influence on the positioning accuracy of the end of the manipulator. The mechanical arm rod requires higher static stiffness and dynamic natural frequency. At the same time, the quality of the arm rod should be minimized as much as possible because the ratio of weight of the arm rod is large relative to other parts. The traditional optimization method is difficult to realize the light weight design while considering both the structural dynamic and the static natural frequency characteristics [6]. In order to give consideration to the higher dynamic static specific and smaller quality requirements of the space robotic manipulator, the multi-constraint topological optimization is used to study the structural lightweight design of the arm of the space manipulator [7].

\section{Space Robotic Manipulator}

This paper studies the six-DOF space robotic manipulator developed independently. According to the six-DOF space robotic manipulator configuration, the mechanism diagram of the manipulator is established [8]. At the same time the D-H coordinate system of the manipulator is set up as shown in Fig.1. 


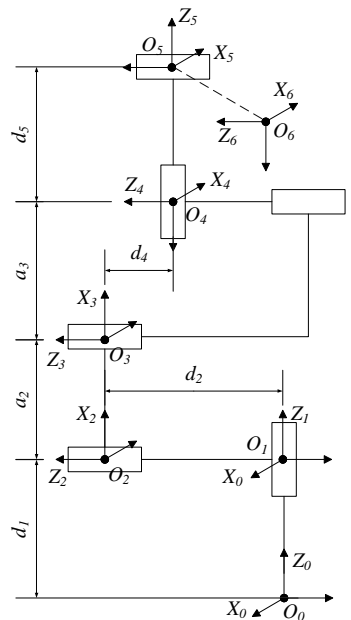

Fig.1 D-H Coordinate System of the Manipulator

The coordinate origin $\{\mathrm{O} 6\}$ of the end of the manipulator is coincident with the coordinate origin $\{\mathrm{O} 5\}$. The D-H parameters of the space robotic manipulator are shown in Table.1.

Table.1 D-H Parameters of the Manipulator

\begin{tabular}{cccccc}
\hline & $\alpha_{i-1}\left({ }^{\circ}\right)$ & $a_{i-1}(\mathrm{~mm})$ & $\theta_{i}\left({ }^{\circ}\right)$ & $d_{i}(\mathrm{~mm})$ & Range of joint \\
\hline 1 & 0 & 0 & $\theta_{1}(0)$ & $d_{1}(123.5)$ & $-180 \sim+180^{\circ}$ \\
2 & 90 & 0 & $\theta_{2}(90)$ & $d_{2}(197.5)$ & $-180 \sim+180^{\circ}$ \\
3 & 0 & $a_{2}(436)$ & $\theta_{3}(0)$ & 0 & $-180 \sim+180^{\circ}$ \\
4 & 0 & $a_{3}(386)$ & $\theta_{4}(90)$ & $-d_{4}(-28.61)$ & $-180 \sim+180^{\circ}$ \\
5 & 90 & 0 & $\theta_{5}(0)$ & $d_{5}(136.39)$ & $-180 \sim+180^{\circ}$ \\
6 & -90 & 0 & $\theta_{6}(0)$ & 0 & $-180 \sim+180^{\circ}$ \\
\hline
\end{tabular}

The working space of the six-DOF space robotic manipulator is analyzed. In the working space, a typical working condition is planned, and dynamic analysis is carried out in ADAMS software. The working load of the large arm rod is calculated, and the maximum load is taken as the load input of optimization topology analysis as shown in figure 2 (a) and (b).

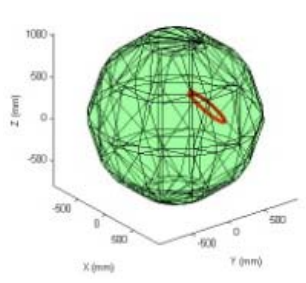

(a)Available Working Space

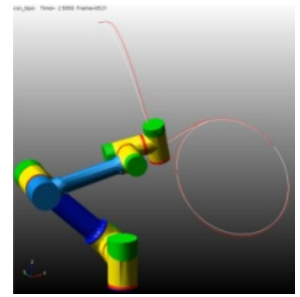

(b) Typical Working Condition Dynamic Analysis

Fig.2 Available Working Space and Typical Working Condition Dynamic Analysis

\section{Multi-Constraint Topology Optimization Mathematical Modeling.}

\subsection{Topology Optimization Design Process}

The structural optimization design process consists of three levels: dimension (or section) optimization, shape (or location) optimization and topology (or layout) optimization. Size optimization and shape optimization have been widely used in practical engineering design, and topological optimization research has been gradually carried out, which has become a hot research field in recent years. Topology optimization is a higher-level approach involving more complex factors, which could solve more complex engineering problems. Topology optimization design involves three factors which consist of design variable, objective function and constraint [9].

Evolutionary structure optimization (ESO) and Bilateral evolutionary structural optimization (BESO) are mainly aimed at two-dimensional or three-dimensional continuum structure. When topology optimization is carried out with the complex three-dimensional continuum, each 
optimization process will be evolved for several times which leads to the amount of data multiplied, and the optimal iteration time long. The structure unit could not be added after deletion with the optimization criterion, or the added unit is not the optimal solution. BESO is based on sensitivity, which could lead to errors in unit selection. In order to improve the optimization speed of ESO and BESO optimization algorithm, the genetic algorithm is adopted to optimize the unit. Genetic bilaterally progressive structural optimization (GBESO) algorithm is based on genetic evolutionary strategy [10]. In order to avoid BESO method to delete some correct units prematurely, the selection mechanism of genetic algorithm is introduced. The removal of the unit is based on the adaptive value, and it is gradually eliminated by probability, avoiding the optimization deviation caused by the BESO quantitative deletion [11-13]. The concrete implementation process is as follows:

1) Design the initial design domain and establish the finite element model

2) The finite element is used as the initial population. The length of the initial chromosome set by each unit is $\mathrm{n}$. Also, the design precision is $\mathrm{n}$. Each gene in the chromosome is initially set to 1

3) The boundary condition and load are added, the finite element analysis is carried out to obtain the structural response data.

4) Calculate the sensitivity of each unit, select the fitness function and calculate the fitness value of the individual.

5) According to the fitness value of the individual, select the replication individual to the next generation population according to the elite retention strategy.

6) The crossover probability is selected to cross the individual and generate new species.

7) The mutation rate was selected to perform mutation operation on individuals with low fitness, and the mutation operator was treated with one-way mutation, which means the gene bit is set to 0 if the bit was not 0 .

8) A unit with chromosome all 0 is removed.

9) If the gene of a unit chromosome is all "1" and its adjacent unit is deleted, it is readded to the deleted unit.

10) The fitness of the individual after the variation is calculated

11) Repeat (3) (10) to satisfy the constraint condition. Obtain the optimal topology structure and terminate the iteration.

The running flow diagram is shown in Fig.3.

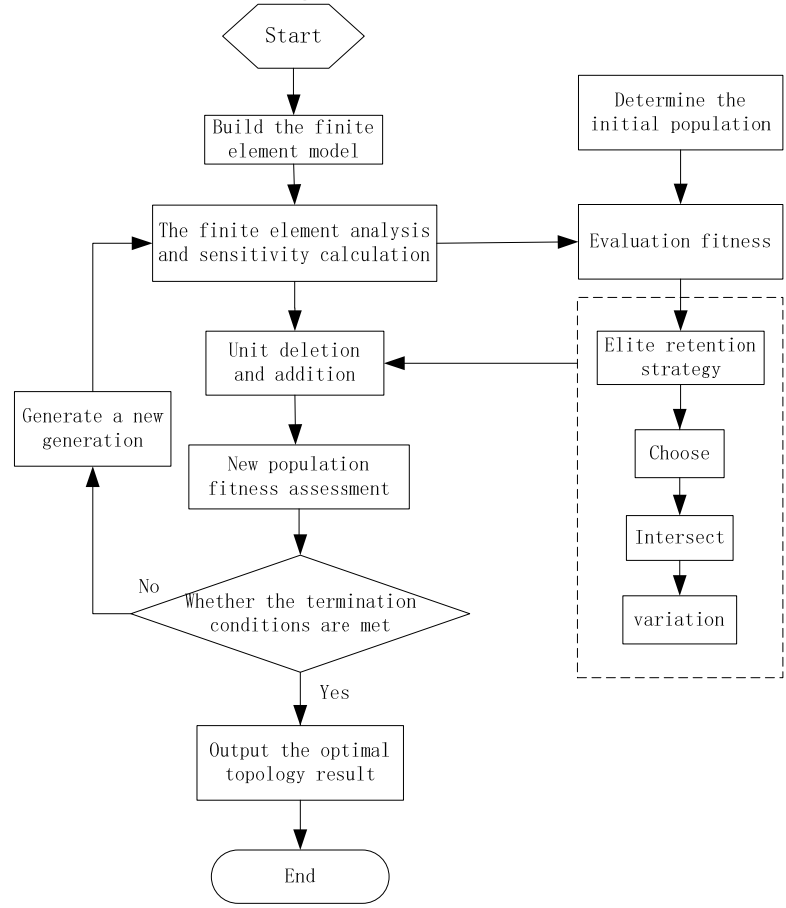

Fig. 3 Flow Diagram of Optimization 


\subsection{Multi - Constraint Topology Optimization Mathematical Modeling}

Under the given load, the mathematic cal model of topology optimization with the constraint condition of stiffness and natural frequency is given.

Find:

$$
\begin{gathered}
d=\left\{d_{1}, d_{2}, \ldots, d_{n}\right\}^{T} \\
\min W=\sum_{i=1}^{n} w_{i} d_{i}
\end{gathered}
$$

s.t.

$$
\begin{gathered}
K>K^{*} \\
\Lambda(d)>\Lambda(d)^{*} \\
\left(K-\lambda_{j}^{2} M\right) \Phi_{j}=0 \\
\Lambda(d)=\sum_{j=1}^{n} \omega_{i}\left(\frac{\lambda_{j}^{\max }-\lambda_{j}(d)}{\lambda_{j}^{\max }}\right)
\end{gathered}
$$

where di is the topology variable of unit $i$ which take 0 and 1 as the value. $W$ is the weight. wi is the weight of unit i. $U$ Is the structural displacement matrix. $K$ is structural stiffness matrix. $u_{e}$ unit displacement vector of unit i. F is the external force vector. $M$ is mass matrix. $\Phi_{j}$ is The j-th mode vector. $\lambda_{j}$ is the j-th natural frequency. $\Lambda(d)$ Is the natural frequency of the structure. $\lambda_{j}(d)$ is the $\mathrm{j}$-th natural frequency function. $\lambda_{j}^{\max }$ is the single target frequency value of the J-th order. $\omega_{i}$ is the weight value of the natural frequency of the $j$ th order.

The stiffness of mechanical system refers to the unit deformation caused by elastomer. The stiffness constraint is transformed into the displacement deformation constraint of the unit. The topology optimization mathematical model could be transformed into the form as below.

s.t.

$$
\begin{gathered}
\varepsilon_{i} \leq \varepsilon^{*} \\
\Lambda(d)>\Lambda(d)^{*} \\
d_{i}=\{0,1\}(\mathrm{i}=1,2 \ldots, \mathrm{n})
\end{gathered}
$$

where $\varepsilon_{i}$ is the strain of the unit and is the allowable strain of the unit.

Due to the deletion of unit $\mathrm{i}$, the strain sensitivity of unit $\mathrm{k}$ is shown as below.

$$
\Delta \varepsilon^{v m}=\sim_{\varepsilon_{k}}^{v m}-\varepsilon_{k}^{v m} k \neq i
$$

Where $\tilde{\varepsilon}_{k}^{v m}$ is the strain of unit $\mathrm{k}$ after the unit $\mathrm{i}$ deleted and $\Delta \varepsilon^{v m}$ is the strain before the unit $\mathrm{i}$ is removed.

\section{Results}

The model is made up of point clouds after topology optimization which could not be directly applied to machining processing. According to the optimization results, the pre-optimization model should be modified manually. The material of the arm rod should be removed as far as possible, while the material of the points cloud position in the optimization model should be retained. The reinforcement characteristics will be added in the corresponding position. The mass of the original space manipulator is $1.71 \mathrm{Kg}$. In contrast the quality of the optimized machining entity model is reduced to $1.10 \mathrm{Kg}$ which is decreased by $35.67 \%$. The structures are shown in Fig.4 (a), (b) and (c) before and after topology optimization. 


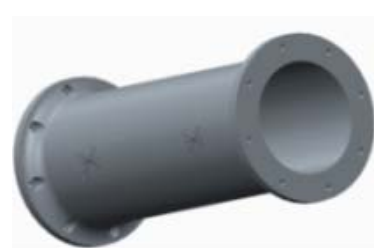

(a)

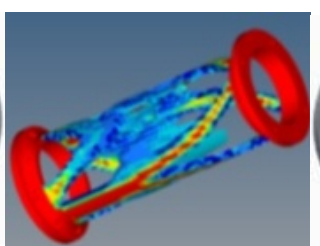

(b)

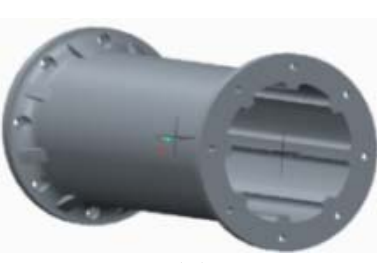

(c)

Fig.4 Structure Before and After Topology Optimization

The natural frequencies before and after optimization are analyzed, as shown in Fig. 6 (a) and (b).

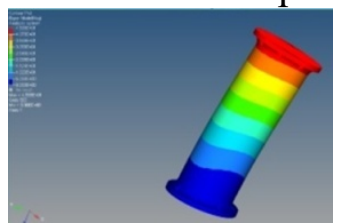

(a)

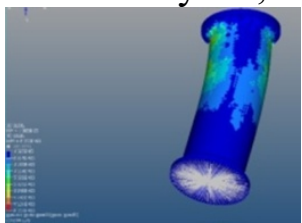

(b)

Fig.5 Natural Frequency Calculation Before and After Optimization

By comparing the natural frequency changes before and after optimization, the natural frequency of the first to third order is obviously improved, while the fourth and fifth order is roughly the same as that before optimization. It could be considered that the natural frequency of the arm rod of the optimized space robotic manipulator is obviously improved, which proves the optimization method is effective. The natural frequencies before and after optimization are listed in Table.2.

Table 2 Comparison of Natural Frequency Before and After Optimization

\begin{tabular}{ccc}
\hline Order of modes & Natural frequency before optimization & Natural frequency after optimization \\
\hline 1 & $9.406 \mathrm{E}+02$ & $1.053 \mathrm{E}+03$ \\
2 & $9.409 \mathrm{E}+02$ & $1.054 \mathrm{E}+03$ \\
3 & $2.631 \mathrm{E}+03$ & $2.758 \mathrm{E}+03$ \\
4 & $3.883 \mathrm{E}+03$ & $3.820 \mathrm{E}+03$ \\
5 & $3.884 \mathrm{E}+03$ & $3.871 \mathrm{E}+03$ \\
\hline
\end{tabular}

The maximum deformation displacement after optimization was reduced from 0.01811 to 0.01752 decreased by $3.25 \%$, as shown in Fig.6 (a) and (b).

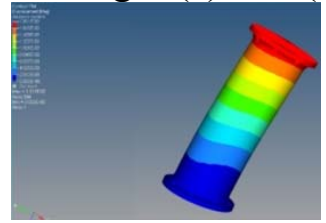

(a)

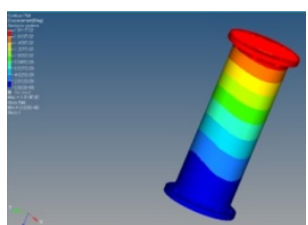

(b)

Fig. 6 The maximum deformation displacement before and after optimization

\section{Conclusion}

It could be used to reduce the weight of the arm rod of the space robotic manipulator as well as improve the stiffness and natural frequency of the structure by the multi-constraint topology optimization. That means the overall performance of the space robotic manipulator could be improved obviously. It proves the effectiveness of multi-constraint topology optimization to the space robotic manipulator by the comparison of the optimizing results. This optimization method could be applied to other structures and mechanism of space robotic manipulator to improve the payload of the end of manipulator, natural frequency and the positioning accuracy fundamentally.

\section{References}

[1]. Haidegger T. Advanced Robotic Arms in Space[C]. The 55th International Astronautical Congress, Vancouver, Canada, 2004: 1-10. 
[2]. James F A, Peter D S. The Development Test Flight of the Flight Telerobotic Servicer: Design Description and Lessons Learned[J]. IEEE Transactions on Robotics and Automation, 1993, 9(3): 664-674.

[3]. Staritz P J, Skaff S, Urmson C. Skyworker: a Robot for Assembly, Inspection and Maintenance of Large Scale Orbital Facilities[C]. IEEE International Conference on Robotics and Automation, Seoul, Korea, 2001: 4180-4185.

[4]. Lovchik C., Diftler M. The Robonaut Hand: A Dexterous Robot Hand for Space[C]. IEEE International Conference on Automation and Robotics, Detroit, Michigan, 1999: 907-912.

[5]. Wilson, J. R. Satellite Hopes Ride on ORBITAL EXPRESS[J]. Aerospace America, 2007, 45(2): $30-35$.

[6]. Xiao ZY, Duan JZ, Du XQ, et al. Finite element static analysis and design improvement for motorman industrial robot[J]. Journal of Changsha University of Science and Technology (Natural Science), 2011, 8(4):71-76(in Chinese).

[7]. Gao DQ, Mao ZY, Zhang GX, et al. Static and dynamic performance analysis and structural improvement of DVG850 workbench[J]. Mechanical Design \& Manufacture, 2011(3):146-147.

[8]. Kazuya Yoshida. Expanding the Horizons of Service and Exploration. IEEE Robotics \& Automation Magazine, 2009,03(10):20-27.

[9]. Wu SF. Study on the topology optimization and model reconstruction of mechanical structure GBESO method based on CAD/CAE rapid response[D]. Shanxi: North university of china, 2014:1-30.

[10]. Rozvany G I N. A critical review of established methods of structural topology optimization[J]. Structural and Multidisciplinary Optimization. 2009,37(3): 217-237.

[11]. Sigmund O. Morphology-based black and white filters for topology optimization[J]. Structural and Multidisciplinary Optimization. 2007,33(4-5): 401-424.

[12]. Lipton S,Evans J A,Bazilevs Y,et al. Robustness of isogeometric structural discretizations under severe mesh distortion[J]. Computer Methods in Applied Mechanics and Engineering. 2010,199(5): 357-373.

[13]. Garcia M J,Gonzalez C A. Shape optimisation of continuum structures via evolution strategies and fixed grid finite element analysis[J]. Structural and Multidisciplinary Optimization. 2004,26(1-2): 92-98. 